\title{
Aplicação do Método Dual-Afim de Pontos Interiores ao Problema de Planicidade de Superfícies
}

\author{
A.R. BALBO ${ }^{1}$, E.C. BAPTISTA ${ }^{2}$, Departamento de Matemática, Universidade \\ Estadual Paulista (UNESP), Av. Eng. Luiz Edmundo C. Coube s/n, 17033-360 \\ Bauru, SP, Brasil
}

M.N. ARENALES ${ }^{3}$, Departamento de Ciências de Computação e Estatística,ICMC, Universidade de São Paulo (USP), Av. Dr. Carlos Botelho 1465, Centro, 13560-970 São Carlos, SP, Brasil.

\begin{abstract}
Resumo. Neste trabalho apresenta-se uma adaptação do Método Dual-Afim de Pontos Interiores e sua aplicação ao Problema de Planicidade de Superfícies, explorado na Engenharia Mecânica, o qual é equivalente ao Problema de Sistemas Lineares Inconsistentes na Norma de Tchebyshev. Testa-se uma implementação computacional da adaptação feita, do Método Dual-Afim, à determinação de soluções aproximadas de Problemas de Planicidade e compara-se com resultados já obtidos e publicados.
\end{abstract}

\section{Introdução}

Os métodos de pontos interiores propostos, entre outros, em [7] e [1], e amplamente investigados em [4], principalmente aqueles inseridos na metodologia DualAfim e Primal-Dual de Pontos Interiores, tem sido muito explorados nestas últimas décadas para solucionar, com eficiência, Problemas de Programação Matemática Linear, Não-Linear e Inteira. Aplicações destes a Problemas de Engenharia tem mostrado sua eficiência. Em Engenharia Mecânica, existe uma área denominada Metrologia de Superfície que se preocupa com a medição dos desvios de uma peça em comparação com uma forma pretendida. Faz parte dessa área o estudo da Planicidade de uma Superfície, o qual é equivalente ao Problema de Sistemas Lineares Inconsistentes na Norma de Tchebyshev, que pode ser expresso como um Problema de Programação Linear e, assim, ser solucionado pela Metodologia de Pontos Interiores citada. Este problema é definido como a menor distância entre dois planos paralelos, denominados Planos de Controle, os quais contém toda a superfície e consiste em aproximar a superfície por um plano, denominado Plano Médio, o qual é paralelo aos Planos de Controle. Vários métodos foram utilizados para resolver o Problema de Planicidade, tais como: o Método Mínima Zona, encontrado em

\footnotetext{
${ }^{1}$ arbalbo@fc.unesp.br; o trabalho do primeiro autor foi parcialmente financiado pela FAPESP.

${ }^{2}$ baptista@fc.unesp.br

3 arenales@icmc.usp.br
} 
[6], uma Especialização do Método Simplex, apresentado em [3] e o Método BitFit Least Square, visto em [8], entre outros. Neste trabalho apresenta-se uma aplicação do Método Dual-Afim de Pontos Interiores para análise do Problema de Planicidade. Adapta-se estes métodos à determinação de soluções aproximadas do problema proposto utilizando-se de uma extensão da implementação computacional encontrada em [2]. Esta implementação é testada nos problemas apresentados em [6] e [3] para comparação de resultados. Inicialmente, na Seção 2, é formulado o Problema de Planicidade como um Problema de Programação Linear (PPL) definido a partir de Sistemas Lineares Inconsistentes na Norma de Tchebyshev. Na Seção 3, faz-se uma análise do Algoritmo Dual-Afim de Pontos Interiores e propõe-se uma primeira modificação deste, visando solucionar o Problema de Planicidade, para Sistemas Lineares Inconsistentes na Norma de Tchebyshev, considerando-se a hipótese de variável não-negativa. Na Seção 4 é então proposta uma extensão do algoritmo citado para tratar Problemas de Planicidade, os quais, na prática são equivalentes ao Problema de Sistemas Lineares Inconsistentes citado, o qual é

definido para variáveis irrestritas. São determinadas estratégias para a resolução e o tratamento destes problemas, considerando-se estas variáveis. Finalmente, na Seção 5 apresenta-se a aplicação de uma implementação do algoritmo proposto em alguns Problemas de Planicidade, comparando-se os resultados obtidos com [6] e [3].

\section{A Definição do Problema de Planicidade}

O Problema de Planicidade pode ser formulado como um Problema de Programação Linear (PPL) definido a partir de um Problema de Sistemas Lineares Inconsistentes na Norma de Tchebyshev, expresso por:

$$
\operatorname{minimizarf}(s)=\|s\|_{\infty} ; \text { sujeito a: } A v+s=b,
$$

onde $A \in R^{m x 3}, v \in R^{3}, b \in R^{m}$ e $s \in R^{m}$.

A matriz de restrições $A=\left(a^{i}\right)$ é definida tal que $a^{i}=(x i, y i, 1)$, para i $=1, \ldots, \mathrm{m}$; onde $(x i, y i) \in R^{2}$, são as coordenadas plotadas no plano $R^{2}$, conhecidas a priori ([6] e [3]). O vetor $b$ é formado pelas medições da superfície, onde $b i=z i, z i \in R^{+}$, tal que $z i$ mede a distância em relação ao ponto $(x i, y i) \in R^{2}$, no espaço $R^{3}$. Assim, os pontos plotados $(x i, y i, z i) \in R^{3}$, conhecidos a priori, definem a superfície em $R^{3}$ a ser contida por dois planos paralelos, denominados Planos de Controle. Estes planos serão obtidos a partir de um Plano Médio e o vetor $v \in R^{3}$ é o vetor de incógnitas contendo as coordenadas deste plano, a serem determinadas pelo método proposto.

De acordo com [5], o PPL dado em (2.1) é expresso de forma equivalente por:

$$
\begin{gathered}
\operatorname{minimizarf}(s)=\|s\|_{\infty}=\max _{1 \leq i \leq m}\left(\left|s_{i}\right|\right)=s_{\text {imax }} \\
\text { sujeito a: }-s_{\text {imax }} \leq s_{i} \leq s_{\text {imax }},
\end{gathered}
$$

onde

i) $s_{i}=b_{i}-a^{i} v$, para $\mathrm{i}=1 \ldots \mathrm{m}$; 
ii) $\mathrm{i}=$ imax tal que

$$
s_{\text {imax }}=\max _{1 \leq i \leq m}\left(\left|s_{i}\right|\right) .
$$

O PPL Dual associado a (2.2), é posto a seguir

$$
\operatorname{maximizarf}(w)=w^{T} b ; \text { sujeito a: } A^{T} w=0 ;\|w\|_{1}=1 ; w \in R^{m} ;
$$

onde $\|w\|_{1}=\sum_{i=1}^{m}\left|w_{i}\right|$. Considerando-se os PPL's definidos acima, será desenvolvida uma extensão do Algoritmo Dual-Afim de Pontos Interiores para resolver o Problema de Planicidade, o qual é equivalente ao PPL (2.1). Para este objetivo, inicialmente são apresentadas, na próxima seção, as idéias básicas do Método Dual-Afim de Pontos Interiores.

\section{Idéias Básicas do Algoritmo Dual-Afim}

O Algoritmo Dual-Afim de pontos interiores inicializa-se com uma solução viável e objetivando a otimalidade do PPL (2.1), diminui progressivamente a Função Objetivo, preservando a factibilidade do conjunto de restrições no processo. Para solucionar o Problema (2.1), inicialmente será desenvolvida uma extensão do Algoritmo Dual-Afim de Pontos Interiores para o caso em que a variável $s$ é não-negativa e em seguida faz-se uma extensão deste algoritmo para o caso (2.1), em que as variáveis são irrestritas. Com esta intenção, reesecreve-se o PPL definido em (2.1) por:

$$
\operatorname{minimizarf}(s)=\|s\|_{\infty} ; \text { sujeito a: } A v+s=b ; s \geq 0 .
$$

\subsection{Direções de Busca}

Para auxiliar na definição das Direções de Busca do Algoritmo a ser definido, redefiniremos a Função Objetivo Primal de forma equivalente por:

$$
f(s)=\|s\|_{\infty}=\max _{1 \leq i \leq m}\left(\left|s_{i}\right|\right)=\left(e^{i}\right)^{T} s
$$

onde $e^{i}=(0, \ldots, 1, \ldots 0)^{T}$ é o versor da base canônica, com valor unitário na i-ésima componente tal que $\mathrm{i}=$ imax (índice que contém o maior valor em módulo de $s_{i}$ ).

Seja na k-ésima iteração a solução interior $\left(v^{k}, s^{k}\right)$ tal que $A v^{k}+s^{k}=b$ e $s^{k}>0$. Objetiva-se encontrar uma boa Direção de Movimento $\left(d_{v}^{k}, d_{s}^{k}\right)$ e o passo $\theta^{k}>0$, a ser dado nesta direção, tal que uma nova solução interior $\left(v^{k+1}, s^{k+1}\right)$ seja gerada:

$$
v^{k+1}=v^{k}+\theta^{k} d_{v}^{k} ; s^{k+1}=s^{k}+\theta^{k} d_{s}^{k}
$$

que satisfaz

$$
A v^{k+1}+s^{k+1}=b e s^{k+1}>0
$$

e, considerando-se (3.2),

$$
\left(e^{i}\right)^{T} s^{k+1} \leq\left(e^{i}\right)^{T} s^{k} .
$$

Considerando-se (3.3) substituída em (3.4), de tal forma que se garanta a factibilidade de $\left(v^{k+1}, s^{k+1}\right)$, então, as Direções de Busca devem satisfazer

$$
A d_{v}^{k}+d_{s}^{k}=0 ; d_{s}^{k}=-A d_{v}^{k} .
$$


Com o objetivo de se minimizar o valor objetivo, substituindo-se (3.3) em (3.5), obtém-se uma outra exigência para a Direção de Busca:

$$
\left(e^{i}\right)^{T} d_{s}^{k} \leq 0
$$

Condicionado à (3.6) e (3.7), a idéia básica do Método Dual-Afim é a de centrar a solução corrente $s^{k}$ em $e=(1, \ldots, 1)^{T}$, em um espaço transformado, tal que, se preserve a não-negatividade das novas variáveis definidas neste espaço. Para isto, define-se uma matriz mudança de escala $S_{k}=\operatorname{diag}\left(s_{i}^{k}\right)$, a qual é uma matriz diagonal, cujo i-ésimo elemento diagonal é $s_{i}^{k}$. Desta maneira $S_{k}^{-1} s^{k}=e$ e cada variável é transformada (ou escalada) em uma nova variável $s$ tal que $u=S_{k}^{-1} s$ e $s=S_{k} u$. Além disso, se $d_{u}^{k}$ for a Direção de Busca no espaço transformado, então sua direção correspondente no espaço original é dada por:

$$
d_{s}^{k}=S_{k} d_{u}^{k}
$$

Afim de se definir uma boa Direção de Busca no Espaço Transformado, preservando a (3.6) tem-se

$$
A d_{v}^{k}+d_{s}^{k}=0 \Longrightarrow A d_{v}^{k}+S_{k} d_{u}^{k}=0 \Longrightarrow S_{k}^{-1} A d_{v}^{k}=-d_{u}^{k} .
$$

Multiplicando-se ambos os lados de (3.9) por $A^{T} S_{k}^{-1}$ encontra-se:

$$
A^{T} S_{k}^{-2} A d_{v}^{k}=-A^{T} S_{k}^{-1} d_{u}^{k}
$$

Assumindo que A é de posto completo $\mathrm{m}, A^{T} S_{k}^{-2} A$ é definida positiva, então obtém-se:

$$
A^{T} S_{k}^{-2} A d_{v}^{k}=-A^{T} S_{k}^{-1} d_{u}^{k}
$$

Denotando-se $Q_{k}=\left(A^{T} S_{k}^{-2} A\right)^{-1} A^{T} S_{k}^{-1}$, a expressão anterior é simplificada por:

$$
d_{v}^{k}=-Q_{k} d_{u}^{k} .
$$

A Equação (3.10) implica que $d_{v}^{k}$ é atualmente determinada por $d u^{k}$ no espaço transformado. Encontrando-se uma direção apropriada tal que (3.7) é satisfeita, então a minimização da Função Objetivo está garantida. Com esta intenção definese

$$
d_{u}^{k}=-Q_{k}^{T} A^{T} e^{i}
$$

Observe que, $d u^{k}=-Q_{k}^{T}\left(a^{i}\right)^{T}$ onde $\left(a^{i}\right)^{T}$ é a i-ésima coluna da matriz A.

Assim, (3.7) está satisfeita, pois

$$
\begin{gathered}
\left(e^{i}\right)^{T} d_{s}^{k}=-\left(e^{i}\right)^{T} A d_{v}^{k}=\left(e^{i}\right)^{T} A Q_{k} d_{u}^{k}= \\
=-\left(e^{i}\right)^{T} A Q_{k} Q_{k}^{T} A^{T} e^{i}=-\left\|\left(e^{i}\right)^{T} A Q_{k}\right\|^{2} \leq 0 .
\end{gathered}
$$

Combinando-se (3.10) e (3.11), chega-se a seguinte Direção de Busca no Espaço Original

$$
d_{v}^{k}=\left(A^{T} S_{k}^{-2} A\right)^{-1} A^{T} e^{i} .
$$


Consequentemente, de (3.6) e (3.13), tem-se:

$$
d_{s}^{k}=-A\left(A^{T} S_{k}^{-2} A\right)^{-1} A^{T} e^{i}
$$

Uma vez que a Direção de Busca $\left(d_{v}^{k}, d_{s}^{k}\right)$ é conhecida, o Comprimento do Passo $\theta^{k}>0$ é determinado pela exigência da não-negatividade de $s^{k}$, assim:

i) Se $d_{s}^{k}=0$, o PPL definido em (3.1) tem um valor da Função Objetivo constante em seu domínio viável e $\left(v^{k}, s^{k}\right)$ é a solução ótima deste PPL;

ii) Se $d_{s}^{k}>0$, então o PPL (3.1) é ilimitado;

iii) Caso contrário:

$$
\theta_{k}=\min _{1 \leq i \leq m}\left\{\frac{-\alpha s_{i}^{k}}{\left(d_{s}^{k}\right)_{i}} \text { tal que }\left(d_{s}^{k}\right)_{i}<0\right\}
$$

onde $0<\alpha<1$.

Note que, considerando-se o PPL Dual definido em (3.3) é possível definir-se o vetor "estimativa dual" para o algoritmo Dual-Afim de pontos interiores, a qual é feita da seguinte forma:

$$
w^{k}=S_{k}^{-2} d_{s}^{k}+e^{i}
$$

pois, desta forma a restrição do Problema Dual (3.3) verifica-se:

$$
A^{T}\left(w^{k}\right)=A^{T} S_{k}^{-2} d_{s}^{k}+A^{T} e^{i}=-\left(A^{T} S_{k}^{-2} A\right) d_{v}^{k}+A^{T} e^{i}=-A^{T} e^{i}+A^{T} e^{i}=0 .
$$

Para atender a restrição $\|w\|_{1}=1$, basta agora definir $w^{k}$ por:

$$
w^{k} \longleftarrow \frac{w^{k}}{\left\|w^{k}\right\|_{1}}
$$

Assim, $w^{k}$ pode ser visto como o vetor "estimativa dua" no Algoritmo Dual-Afim se é solução do PPL (3.3). Com as soluções $w^{k}$ e $s^{k}$, relativas, respectivamente, aos Problemas (3.1) e (3.3), é possível calcular a folga de dualidade através de $\left(e^{i}\right)^{T} s^{k}-b^{T} w^{k}$. Além disso, se $\left(e^{i}\right)^{T} s^{k}-b^{T} w^{k}=0$, então $\left(v^{k}, s^{k}\right)$ é a solução ótima para (3.1) e $w^{k}$ é a solução ótima para (3.3). Esta informação pode ser utilizada para definir o critério de parada do Algoritmo Dual-Afim de Pontos Interiores e explorada posteriormente no Problema de Planicidade. Isto é feito de forma aproximada por $\left(e^{i}\right)^{T} s^{k}-b^{T} w^{k} \leq \epsilon$, com $\epsilon>0$ uma pequena tolerância positiva.

\subsection{Algoritmo Dual-Afim}

Baseado nas idéias básicas discutidas na seção anterior, é definido a seguir os passos do algoritmo Afim-Dual para o PPL definido em (3.1).

Passo 1 - (Inicialização): Faça $\mathrm{k}=0$. Dada a solução inicial $\left(v^{0}, s^{0}\right)$ tal que:

$$
A v^{0}+s^{0}=b \text { e } s^{0}>0 .
$$

Passo 2 - (Obter as Direções de Busca): Faça $S_{k}=\operatorname{diag}\left(s_{i}^{k}\right)$ e calcule:

$$
d_{v}^{k}=-\left(A^{T} S_{k}^{-2} A\right)^{-1} A^{T} e^{i}
$$




$$
d_{s}^{k}=-A d_{v}^{k}
$$

Passo 3 - (Teste para Ilimitariedade): Se $d_{s}^{k}=0$, então PARE pois $\left(v^{k}, s^{k}\right)$ é ótimo primal. Se $d_{s}^{k}>0$, então PARE pois o Problema (3.1) é ilimitado.

Passo 4 - (Cálculo da Estimativa Dual): Calcular o vetor estimativa dual:

$$
w^{k}=S_{k}^{-2} d_{s}^{k}+e^{i}
$$

e redefinir $w^{k}$ da mesma forma que em 3.18.

Passo 5 - (Teste de otimalidade): Se $\left(e^{i}\right)^{T} s^{k}-b^{T} w^{k} \leq \epsilon$, onde $\epsilon$ é um número positivo pequeno pré-fixado, então PARE pois $\left(v^{k}, s^{k}\right)$ é ótimo do PPL (3.1) e $w^{k}$ é ótimo do PPL (3.3). Caso contrário, vá para o passo seguinte.

Passo 6 - (Cálculo do Comprimento do Passo): Calcule o Comprimento do Passo:

$$
\theta_{k}=\min _{1 \leq i \leq m}\left\{\frac{-\alpha s_{i}^{k}}{\left(d_{s}^{k}\right)_{i}} \text { tal que }\left(d_{s}^{k}\right)_{i}<0\right\} .
$$

Passo 7 - (Determinação da Nova Solução): Efetue a atualização:

$$
\begin{gathered}
v^{k+1}=v^{k}+\theta^{k} d_{v}^{k} ; \\
s^{k+1}=s^{k}+\theta^{k} d_{s}^{k} .
\end{gathered}
$$

Faça $k \longleftarrow k+1$ e vá para o passo 2 .

\section{Extensão do Algorimo Dual-Afim para o Pro- blema de Planicidade}

O objetivo agora é estender o algoritmo analisado na Seção 3 para o caso em que $s \in R^{m}$ é irrestrita. Reconsiderando o PPL (2.2), é possível reescrevê-lo como um Problema de Restrições Canalizadas visto a seguir:

$$
\operatorname{minimizarf}(s)=\|s\|_{\infty}=\max _{1 \leq i \leq m}\left(\left|s_{i}\right|\right) ; \text { sujeito a: }-e s_{\text {imax }} \leq s \leq e s_{\text {imax }} ;
$$

onde $s=b-A v$ e $e=(1, \ldots, 1)^{T}$.

O PPL Dual de (4.1) é aquele definido em (2.3).

Uma extensão do Algoritmo Dual-Afim, definido na Seção 3, pode ser feita fazendo-se algumas considerações.

Desde que $s \in R^{m}$ é irrestrita, considera-se então a seguinte partição para o vetor $s$ :

$$
s=\left(\begin{array}{c}
s^{p} \\
s^{q}
\end{array}\right) \text { tal que } s^{p} \geq 0, s^{q} \leq 0 \text { e } p+q=m .
$$

Então, afim de se estender a idéia do algoritmo anterior, pode-se reescrever $f(s)$, analogamente a (3.2) por:

$$
f(s)=\|s\|_{\infty}=\max _{1 \leq i \leq m}\left(\left|s_{i}\right|\right)=\left\{\begin{array}{ccc}
\left(e^{i}\right)^{T} s & \text { se } & i \in P \\
-\left(e^{i}\right)^{T} s & \text { se } & i \in Q
\end{array} ;\right.
$$


onde $i=$ imax pertence a um dos conjuntos $P$ e $Q$ definidos a seguir:

$$
P=\left\{i \text { tal que } s_{i}>0\right\} ; Q=\left\{i \text { tal que } s_{i}<0\right\} .
$$

O PPL (4.1) pode ser redefinindo por:

$$
\operatorname{minimizarf}(s)=\|s\|_{\infty}=\max _{1 \leq i \leq m}\left(\left|s_{i}\right|\right)=s_{\text {imax }} ; \text { sujeito a: } d \leq s \leq f ;
$$

onde $d=-e s_{\text {imax }} ; f=e s_{\text {imax }}$.

Note que, neste caso, os limitantes, inferior e superior, $d$ e $f$ mudam a cada iteração do método pois dependem de $s_{\text {imax }}$.

Definindo a variável $s$ como a imagem da transformação linear $s=T(v)=b-A v$, tal que, $s$ pertence ao hipercubo $d \leq s \leq f$, então, para fazer a translação de $s$ para um espaço ou hipercubo transformado, necessitam-se de alguns cuidados na definição da matriz diagonal $S_{k}$. Assim, define-se uma matriz mudança de escala que leva em consideração se a componente $s_{i}^{k}$ está mais próxima da fronteira $d_{i}^{k}$ ou de $f_{i}^{k}$, tal que, $S_{k}=\operatorname{diag}\left(g_{i}^{k}\right)$, onde $g_{i}^{k}$ é definido por:

$$
g_{i}^{k}=\min \left\{b_{i}-a^{i} v^{k}-d_{i}^{k}, f_{i}^{k}-b_{i}+a^{i} v^{k}\right\} .
$$

A definição direta de $g_{i}^{k}$ por (4.5) não é interessante pois para $i=i$ max teríamos o valor de $g_{i}^{k}=0$, o que inviabilizaria a definição da matriz diagonal $S_{k}$.

Assim, é interessante explorar as particularidades de (4.1) considerando-se os seguintes casos para a definição de $g_{i}^{k}$ :

i) se i $\in P$, então, $s_{i}^{k} \geq 0$ e o valor mínimo de $g_{i}^{k}$ em (4.5) ocorre em $f_{i}^{k}-b_{i}+a^{i} v^{k}$.

Desde que, $f_{i}^{k}=s_{i \max }^{k}$, então, $0 \leq b_{i}-a^{i} v^{k} \leq s_{\text {imax }}^{k}$

ii) se i $\in Q$, então, $s_{i}^{k} \leq 0$ e o valor mínimo de $g_{i}^{k}$ em (4.5) ocorre em $b_{i}-a^{i} v^{k}-d_{i}^{k}$.

Desde que, $d_{i}^{k}=-s_{i \max }^{k}$, então, $0 \leq-b_{i}+a^{i} v^{k} \leq s_{i \max }^{k}$.

Com estas considerações, pode-se definir os elementos diagonais da matriz $S_{k}$, de forma explícita, por:

$$
g_{i}^{k}=\left\{\begin{array}{lll}
b_{i}-a^{i} v^{k} & \text { se } & i \in P \\
a^{i} v^{k}-b_{i} & \text { se } & i \in Q
\end{array} .\right.
$$

Assim, cada variável $s$ é transformada (ou escalada) em uma nova variável $u \in R^{m}$ tal que $u=S_{k}^{-1} s$ pertence ao hipercubo $S_{k}^{-1} d \leq u \leq S_{k}^{-1} f$.

Seguindo o que foi feito de (3.8) a (3.13), devido à (4.3), a Direção de Busca $d_{v}^{k}$ deve ser redefinida da seguinte maneira:

$$
d_{v}^{k}=\left\{\begin{array}{ccc}
\left(A^{T} S_{k}^{-2} A\right)^{-1} A^{T} e^{i} & \text { se } & i \in P \\
-\left(A^{T} S_{k}^{-2} A\right)^{-1} A^{T} e^{i} & \text { se } & i \in Q
\end{array}\right.
$$

tal que $d_{s}^{k}=-A d_{v}^{k}$.

A definição de $d_{v}^{k}$ em (4.7) influencia diretamente na determinação do vetor estimativa dual $w^{k}$ para o PPL (2.3), o qual tem definição análoga à (3.16), expresso por:

$$
w^{k}=\left\{\begin{array}{lll}
S_{k}^{-2} d_{s}^{k}+e^{i} & \text { se } & i \in P \\
S_{k}^{-2} d_{s}^{k}-e^{i} & \text { se } & i \in Q
\end{array},\right.
$$


para em seguida definí-lo da mesma forma que em (3.18).

O tamanho do passo em uma determinada direção é definido a partir de:

$$
\begin{gathered}
\theta_{k}=\min \left\{\theta_{k}^{1}, \theta_{k}^{2}\right\}, \quad \text { tal que } \\
\theta_{k}^{1}=\min _{1 \leq i \leq m}\left\{\frac{-\alpha s_{i}^{k}}{\left(d_{s}^{k}\right)_{i}} \text { tal que }\left(d_{s}^{k}\right)_{i}<0, \text { se } i \in P\right\}, \\
\theta_{k}^{2}=\min _{1 \leq i \leq m}\left\{\frac{\alpha s_{i}^{k}}{\left(d_{s}^{k}\right)_{i}} \text { tal que }\left(d_{s}^{k}\right)_{i}>0, \text { se } i \in Q\right\} .
\end{gathered}
$$

Assim, com as redefinições de $f(s)$ em (4.2), da matriz $S_{k}$ em (4.6), da Direção de Busca $d_{v}^{k}$ em (4.7), do vetor estimativa dual $w^{k}$ em (4.8) e do tamanho do passo $\theta_{k}$, dado em uma determinada direção, visto em (4.9) ou (4.10), um algoritmo análogo àquele visto na Seção 3.2 pode ser definido para o Problema de Planicidade (2.1), o qual, segue os mesmos passos do Algoritmo Dual-Afim visto naquela seção.

\section{Aplicação do Algoritmo em Problemas de Plani- cidade}

As medições das duas superfícies apresentadas a seguir, encontradas em [6], foram testadas computacionalmente em [6] e [3]. Neste artigo, para a determinação de suas planicidades, utilizou-se uma implementação computacional do algoritmo visto na Seção 4, em linguagem Pascal 7.0, para comparação de resultados. Considerou-se o valor de $\epsilon$ da ordem de $\left(10^{-3}\right)$, para critério de parada e $\epsilon_{1}=10^{-5}$ para controle de atualização de direções e de novas soluções. Apresentam-se as Tabelas 1 e 3, as quais contém as Medições da Superfície e as Tabelas 2 e 4 que mostram os resultados obtidos. Nas Tabelas 2 e 4 destacam-se os seguintes resultados: a inclinação do Plano Médio (solução), a verificação da restrição dual $A^{T} w=0$, a avaliação da folga complementar (folga) e a distância mínima obtida entre os Planos de Controle (planicidade $=2 s_{\text {imax }}$ ), calculada pelos quatro métodos investigados (Método Dual-Afim de Pontos Interiores (MDPI), Especialização do Método Simplex (EMSimplex), Método de Mínima Zona (MMZ) e Método dos Quadrados Mínimos (QMínimos)). Destaca-se que os resultados obtidos pelo Método EMSimplex, encontrado em [3] e pelo Método Mínima Zona (MMZ), encontrado em [6], têm valores muito próximos. Por isso os resultados apresentados pelo EMSimplex não são apresentados neste trabalho.

\subsection{Problema de Planicidade 1}

Tabela 1 - Medições da Superfície 1

\begin{tabular}{|c|c|c|c|c|c|c|c|c|c|}
\hline$x_{i}$ & 0.0 & 1.0 & 2.0 & 0.0 & 1.0 & 2.0 & 0.0 & 1.0 & 2.0 \\
$y_{i}$ & 0.0 & 0.0 & 0.0 & 1.0 & 1.0 & 1.0 & 2.0 & 2.0 & 2.0 \\
$z_{i}$ & 6.9 & 6.9 & 0.0 & -2.5 & -0.5 & -1.0 & 0.0 & 15.7 & 6.9 \\
\hline
\end{tabular}


Tabela 2 - Comparação dos Resultados

\begin{tabular}{|c|c|c|c|}
\hline solucao & $A^{T} w$ & Folga & Planicidade \\
1.360 & $8.0 x 10^{-5}$ & $9.0 x 10^{-3}$ & $M D P I=14.34$ \\
3.710 & $-1 \times 10^{-5}$ & $*$ & $M M Z=14.34$ \\
-0.26 & $-1.3 \times 10^{-5}$ & $*$ & QMinimos $=16.48$ \\
\hline
\end{tabular}

\subsection{Problema de Planicidade 2}

Tabela 3 - Medições da Superfície 2

\begin{tabular}{|l|l|l|l|l|l|l|l|l|l|l|l|l|l|l|l|l|l|l|l|l|}
\hline$x_{i}$ & 0 & 1 & 2 & 3 & 4 & 0 & 1 & 2 & 3 & 4 & 0 & 1 & 2 & 3 & 4 & 0 & 1 & 2 & 3 & 4 \\
& 0 & 1 & 2 & 3 & 4 & & & & & & & & & & & & & & & \\
$y_{i}$ & 0 & 0 & 0 & 0 & 0 & 1 & 1 & 1 & 1 & 1 & 2 & 2 & 2 & 2 & 2 & 3 & 3 & 3 & 3 & 3 \\
& 4 & 4 & 4 & 4 & 4 & & & & & & & & & & & & & & & \\
$z_{i}$ & 2 & 5 & 6 & 8 & 9 & 5 & 7 & 8 & 9 & 12 & 6 & 7 & 8 & 9 & 11 & 7 & 7 & 6 & 7 & 9 \\
& 7 & 6 & 6 & 6 & 8 & & & & & & & & & & & & & & & \\
\hline
\end{tabular}

Tabela 4 - Comparação dos Resultados

\begin{tabular}{|c|c|c|c|}
\hline solucao & $A^{T} w$ & Folga & Planicidade \\
1.270 & $6.27 x 10^{-6}$ & $6.8 \times 10^{-5}$ & $M D P I=4.86$ \\
0.046 & $-1.5 x 10^{-6}$ & $*$ & $M M Z=4.86$ \\
4.430 & $-0.945 x 10^{-6}$ & $*$ & QMinimos $=5.9$ \\
\hline
\end{tabular}

\section{Conclusão}

Neste trabalho fez-se uma adaptação do Método Dual-Afim de Pontos Interiores para análise de Problemas de Planicidade, os quais são equivalentes ao Problema de Sistemas Lineares Inconsistentes na Norma de Tchebyshev. Uma implementação do algoritmo foi feita e aplicada em exemplos destes problemas, obtendo-se soluções cujos valores da função objetivo são muito próximos daqueles encontrados em [6] e [3]. Notou-se que, para a convergência do Algoritmo de Pontos Interiores implementado, um número maior de iterações foi realizado em relação aos métodos utilizados pelos autores citados. Isto ocorre devido a alternância de valores máximos encontrados no vetor solução $s_{\text {imax }}$, que provoca um efeito nas soluções denominado de "efeito planador". Este efeito influencia na estabilidade e convergência do algoritmo, devido às oscilações nas inclinações do Plano Médio procurado, a cada valor de $s_{\text {imax }}$ determinado. Isto exige que o critério de parada definido no Passo 6, do algoritmo visto na Seção 5, seja sensível à determinação da solução ótima, de acordo com a precisão $\epsilon>0$ pré-estimada. Apesar deste fato, obteve-se sucesso com os resultados obtidos pelo Método DAIP, comparados com aqueles encontrados em [6] e [3] e estes incentivam a continuidade e busca de melhoria na implementação feita para se investigar novos e melhores resultados.

Abstract. In this work it is presented an adaptation and extension of the Interior Point Affine-Dual Method and its application to the Surfaces Flatness Problem, ex- 
plored in the Mechanic Engineering, which is equivalent to the inconsistent system problem in the Tchebyshev's norm. A computational implementation of this adaptation it is tested to determinate approximate solutions of the Flatness Problem and it is compared with results already obtained and published.

\section{Referências}

[1] I. Adler, N. Karmarkar, M. Resende e G.Veiga, An implementation of Karmakar's algorithm for linear programming, Mathematical Programming, 12 (1989), 297-335.

[2] A.R. Balbo "Métodos de Pontos Interiores com Mudança de Escala para Programação Linear", Dissertação de Mestrado, ICMSC/USP, São Carlos, 1991.

[3] E.C. Baptista "Sistemas Lineares: Uma Solução na Norma Tchebyshev", Dissertação de Mestrado, ICMSC/USP, São Carlos, 1993.

[4] S.C. Fang e S. Puthenpura, "Linear Optimization and Extensions: Theory and Algorithms", vol. 1, Pretice-Hall, Englewood Cliffs, New Jersey, 1993.

[5] A.P. Francelin, "Aproximação da Solução de Sistemas Lineares Inconsistentes na Norma de Tchebyshev", Dissertação de Mestrado, ICMSC/USP, São Carlos, 1986.

[6] S.T. Huang, K.C. Fan e J.H. Wu, A new minimum zone method for evaluating flatness errors, Precision Engineering, 15 (1993), 25-32.

[7] N. Karmarkar, A new polynomial-time algorithm for linear programming. Combinatorics Engineering, 4 (1984), 373-395.

[8] D.J. Whitehouse. "Handbook of Surface Metrology", Technal Report, Philadelphia Institute of Physics, Philadelphia, 1994. 